\title{
Pulmonary sarcoidosis presenting as a solitary nodule mimicking lung cancer
}

\author{
Tomoyo Taketa ${ }^{1}$ and Takahito Nakamura ${ }^{1}$ \\ ${ }^{1}$ Hoshigaoka Iryo Center
}

February 3, 2021

\begin{abstract}
Sarcoidosis shows various clinical and radiologic manifestations. Nodular sarcoidosis is an uncommon form of pulmonary sarcoidosis. Cases presenting as solid nodules are especially rare. We herein present a case of nodular sarcoidosis with a solid nodule that was suspected to be lung cancer.

Clinical Picture

Pulmonary sarcoidosis presenting as a solitary nodule mimicking lung cancer

Tomoyo Taketa, Takahito Nakamura

Department of Respiratory Medicine, Hoshigaoka Medical Center, Osaka, Japan.

Corresponding author:

Department of Respiratory Medicine, Hoshigaoka Medical Center, Osaka, Japan.

Tomoyo Taketa

tomoyotaketa0623@gmail.com
\end{abstract}

Keywords: sarcoidosis, solitary nodule, lung cancer

Abstract

Sarcoidosis shows various clinical and radiologic manifestations. Nodular sarcoidosis is an uncommon form of pulmonary sarcoidosis. Cases presenting as solid nodules are especially rare. We herein present a case of nodular sarcoidosis with a solid nodule that was suspected to be lung cancer.

A 67-year-old woman with no significant medical history was referred to our hospital for the evaluation of an abnormal shadow on the chest radiograph. The patient was a non-smoker. Physical examination revealed no acute distress. Laboratory tests showed mildly elevated C-reactive protein levels. Lung cancer tumor markers and angiotensin-converting enzyme were within the normal range. Computed tomography (CT) showed a solitary nodule with spicula in the $\mathrm{S}^{6}$ of the right lung (Figure1). Positron emission tomography $(\mathrm{PET}) / \mathrm{CT}$ scan showed mild fluorodeoxyglucose uptake in the nodule and the right hilar lymph node in the delay phase (Figure2). Transbronchial biopsy of specimens demonstrated no malignant findings. We performed video-assisted thoracoscopic lung surgery for the nodule in the right lower lobe, which revealed noncaseating granulomas consistent with sarcoidosis (Figure3). Acid-fast bacilli stain and tissue cultures were negative. Histologic findings established a diagnosis of sarcoidosis.

Nodular sarcoidosis is an uncommon form of sarcoidosis prevalent in $2.4-4 \%$ of cases. ${ }^{1)}$ Most patients present with multiple lung nodules, and solitary lung nodule is a rare radiologic finding in about $18 \%$ of 
nodular sarcoidosis ${ }^{1}$. It is difficult to differentiate between malignancy and nodular sarcoidosis based on radiologic findings. Histological examination is necessary to establish an accurate diagnosis.

\section{ACKNOWLEDGMENTS}

None.

\section{CONFLICT OF INTEREST}

None declared.

\section{AUTHOR CONTRIBUTION}

TT: contributed to write-up of abstract, case presentation and images.

TN; reviewed and edited the manuscript.

\section{ETHICS APPROVAL AND CONSENT TO PARTICIPATE}

Ehics approval was not necessary for this case report. All patient's data and images are de-identified.

INFORMED CONSENT

Written informed consent was obtained from the patient for publication of this case report .

References

Malaisamy S, Dalal B, Bimenyuy C, Soubani A. Clinical and radiological features of nodular pulmonary sarcoidosis. Lung 2009;187:9-15

Figure Legends

Fig1

Chest CT shows a solitary nodule with spicula measuring $3 \mathrm{~cm} \times 2 \mathrm{~cm}$ in the $\mathrm{S}^{6}$ of the right lung.

Fig2

PET scan exhibits fluorodeoxyglucose uptake in the right lower lobe nodule (maximum standardized uptake value (SUVmax) 3.8) and the right hilar lymph node (SUVmax3.6) in the delay phase.

Fig3

Pathological examination of the specimen using thoracoscopic lung surgery reveals non-caseating granulomas.

(Hematoxylin and eosin stain, $\times 100(a) \times 400(b)$ ) 


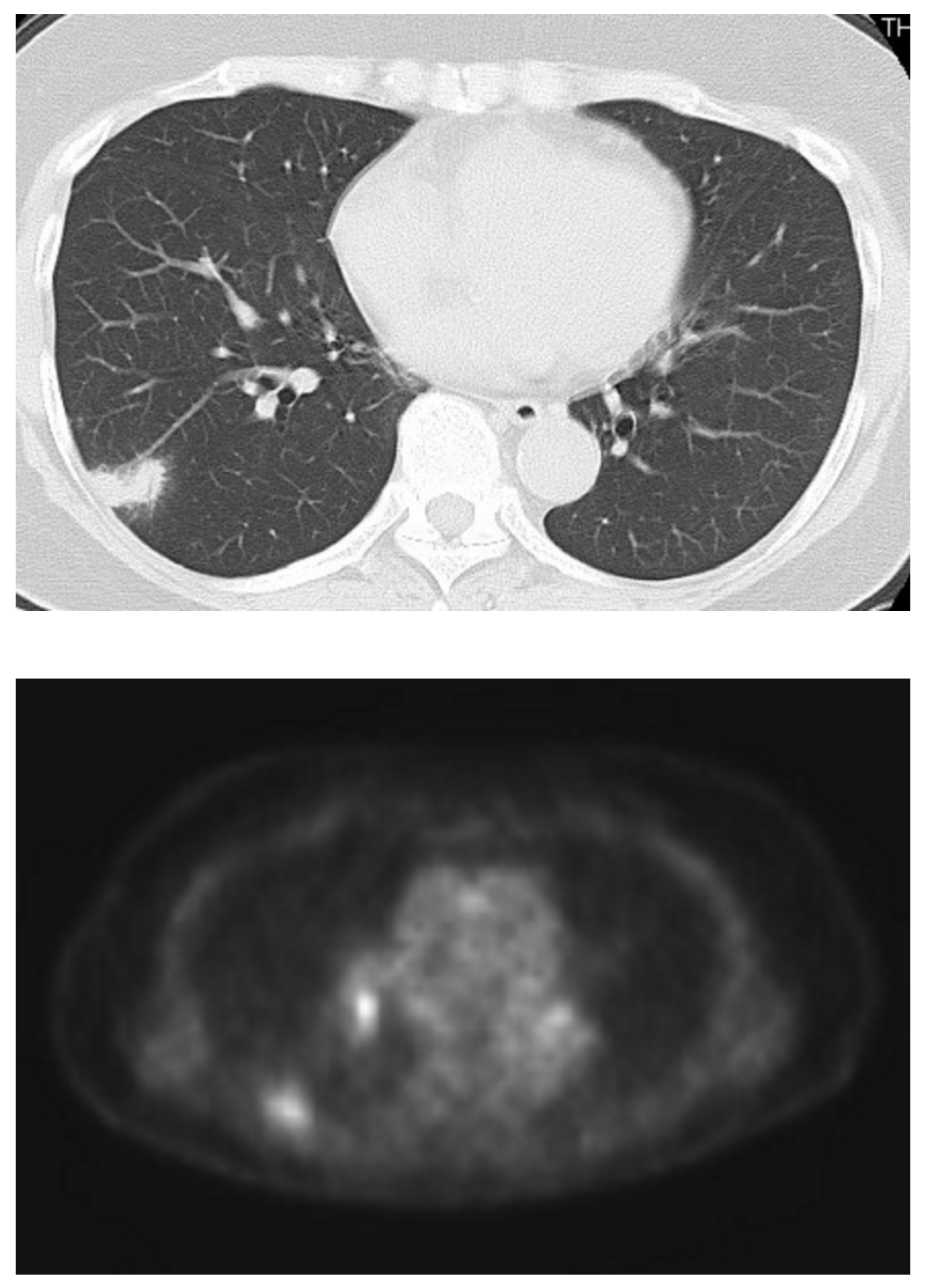



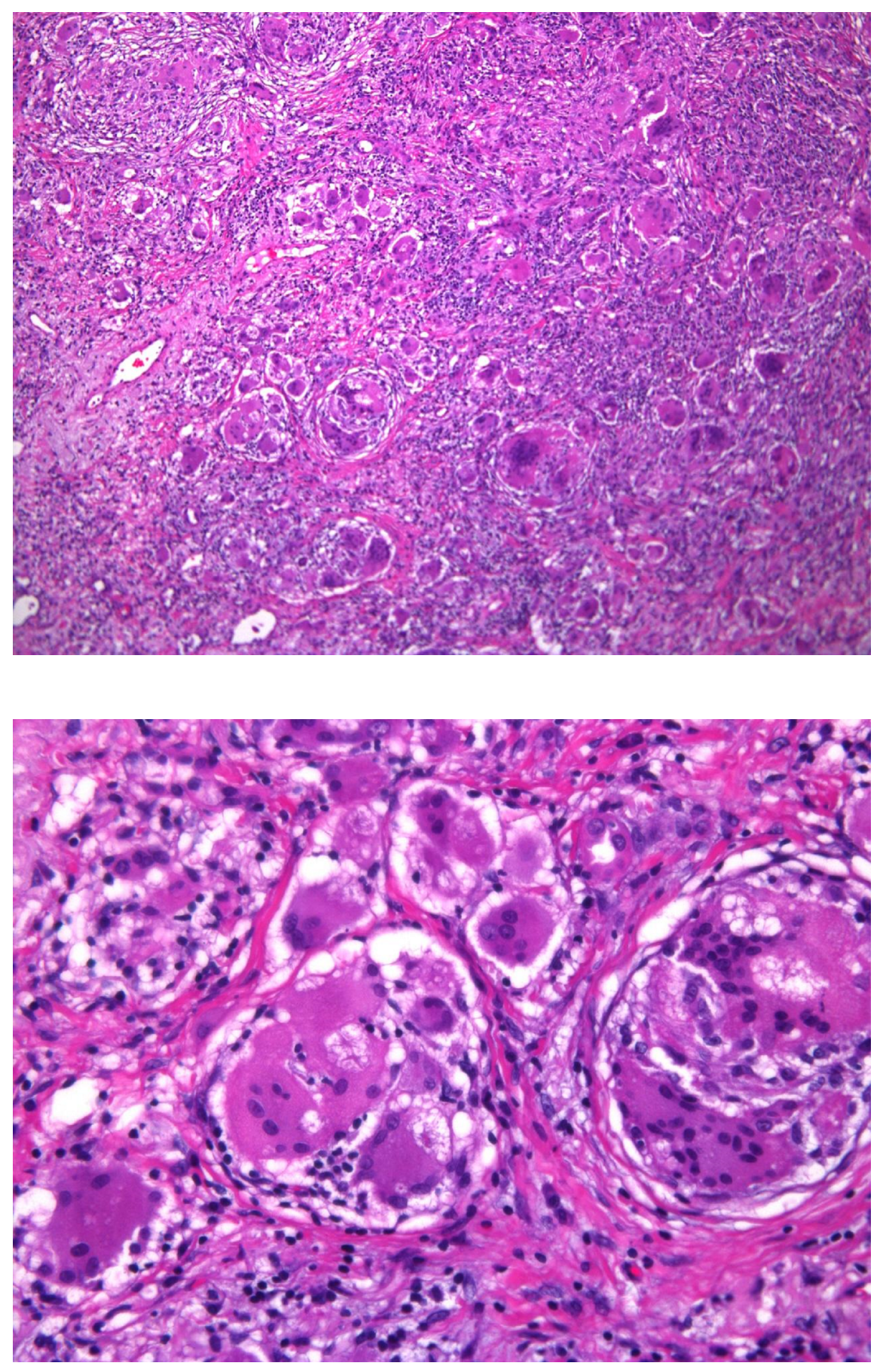